\title{
MEASURE FOR DEPARTURE FROM CUMULATIVE PARTIAL SYMMETRY FOR SQUARE CONTINGENCY TABLES WITH ORDERED CATEGORIES
}

\section{YUSUKE SAIGUSA ${ }^{1}$, MITSUHIRO TAKAMI ${ }^{2}$, AKI ISHII ${ }^{2}$, TOMOYUKI NAKAGAWA ${ }^{2}$ and SADAO TOMIZAWA ${ }^{2}$}

${ }^{1}$ Department of Biostatistics

Yokohama City University School of Medicine

Yokohama City

Kanagawa, 236-0004

Japan

e-mail: saigusay@yokohama-cu.ac.jp

${ }^{2}$ Department of Information Sciences

Faculty of Science and Technology

Tokyo University of Science

Noda City, Chiba, 278-8510

Japan

e-mail: mituhiro291910.t@gmail.com

a.ishii@rs.tus.ac.jp

t_nakagawa@rs.tus.ac.jp

tomizawa@is.noda.tus.ac.jp

2010 Mathematics Subject Classification: 62H17.

Keywords and phrases: cumulative partial symmetry, diversity index, measure, ordered category.

Received March 7, 2019

(C) 2019 Scientific Advances Publishers 


\begin{abstract}
For square contingency tables, Saigusa et al. [7] considered the partial symmetry model which indicates symmetry of cell probabilities for at least one of pairs of symmetric cells with respect to the main diagonal of the table. The present paper proposes (1) another partial symmetry model for symmetric pairs of cumulative probabilities for the square table, and (2) a measure to represent the degree of departure from the model. The measure has a form of weighted geometric mean of the diversity index including Shannon entropy in a special case. Examples are given.
\end{abstract}

\title{
1. Introduction
}

Consider an $r \times r$ square contingency table with same row and column classifications. Let $p_{i j}$ denote the probability that an observation will fall in $i$-th row and $j$-th column of the table $(i=1, \ldots, r ; j=1, \ldots, r)$. Bowker [3] considered the symmetry (S) model defined by

$$
p_{i j}=\psi_{i j} \quad(i=1, \ldots, r ; j=1, \ldots, r),
$$

where $\psi_{i j}=\psi_{j i}$. This model indicates complete symmetry of cell probabilities $\left\{p_{i j}\right\}$ (also see Bishop et al. [2], p. 282).

Saigusa et al. [7] proposed the partial symmetry (PS) model defined by

$$
p_{i j}=\psi_{i j} \quad(i=1, \ldots, r ; j=1, \ldots, r),
$$

where $\psi_{s t}=\psi_{t s}$ for at least one $(s, t)$ with $s \neq t$. This model states that the cell probability that an observation will fall in row category $s$ and column category $t(>s)$ is equal to the probability that the observation falls in row category $t$ and column category $s$ for at least one $(s, t), s \neq t$.

Let $X$ and $Y$ denote the row and column variables, respectively. Let $G_{i j}$ denote the cumulative probability from upper-right or lower-left corner of the $r \times r$ table, i.e.,

$$
G_{i j}=P(X \leq i, Y \geq j)=\sum_{k=1}^{i} \sum_{l=j}^{r} p_{k l} \quad(i<j),
$$


and

$$
G_{i j}=P(X \geq i, Y \leq j)=\sum_{k=i}^{r} \sum_{l=1}^{j} p_{k l} \quad(i>j) .
$$

The $\mathrm{S}$ model can be expressed by

$$
G_{i j}=\Psi_{i j} \quad(i=1, \ldots, r ; j=1, \ldots, r ; i \neq j),
$$

where $\Psi_{i j}=\Psi_{j i}$. Therefore, the $\mathrm{S}$ model also indicates complete symmetry of cumulative probabilities $\left\{G_{i j}\right\}$.

We are now interested in the PS model with the cell probabilities $\left\{p_{i j}\right\}$ replaced by the cumulative probabilities $\left\{G_{i j}\right\}$.

When we analyze data, if a model fits the data poorly, we may be interested in seeing the degree of departure from the model. Tomizawa et al. [9] and Tomizawa et al. [8] proposed measures to express the degree of departure from the S model. Saigusa et al. [7] proposed the measure for the PS model. Assuming $\left\{p_{i j}+p_{j i} \neq 0\right\}$, the measure in Saigusa et al. [7] is defined by

$$
\Phi^{(\lambda)}=\prod_{i=1}^{r-1} \prod_{j=i+1}^{r}\left[\Phi_{i j}^{(\lambda)}\right]^{\left(p_{i j}^{*}+p_{j i}^{*}\right)} \quad(\lambda>-1),
$$

where

$$
\begin{aligned}
p_{i j}^{*} & =\frac{p_{i j}}{\delta}, \quad \delta=\sum_{s \neq t} p_{s t}, \\
\Phi_{i j}^{(\lambda)} & =1-\frac{\lambda 2^{\lambda}}{2^{\lambda}-1} H_{i j}^{(\lambda)}, \\
H_{i j}^{(\lambda)} & =\frac{1}{\lambda}\left(1-\left(p_{i j}^{c}\right)^{\lambda+1}-\left(p_{j i}^{c}\right)^{\lambda+1}\right), \\
p_{i j}^{c} & =\frac{p_{i j}}{p_{i j}+p_{j i}},
\end{aligned}
$$


and the value at $\lambda=0$ is taken to be the limit as $\lambda \rightarrow 0$. This measure is formed by the weighted geometric mean of Patil and Taillie's [6] diversity indices $\left\{H_{i j}^{(\lambda)}\right\}$.

We are also interested in measuring the degree of departure from PS of cumulative probabilities $\left\{G_{i j}\right\}$ instead of cell probabilities $\left\{p_{i j}\right\}$.

This paper proposes (1) the PS model for cumulative probabilities and (2) the measure to represent the degree of departure from the model in Section 2 .

\section{Model and Measure}

\subsection{Model}

For an $r \times r$ contingency table with ordered categories, we propose the model defined by

$$
G_{i j}=\Psi_{i j} \quad(i=1, \ldots, r ; j=1, \ldots, r ; i \neq j),
$$

where $\Psi_{s t}=\Psi_{t s}$ for at least one $(s, t)$ with $s \neq t$. We shall refer to this model as the cumulative partial symmetry (CPS) model. This model states that the probability that an observation will fall in row category $s$ or below and column category $t(>s)$ or above is equal to the probability that the observation falls in row category $t$ or above and column category $s$ or below for at least one $(s, t), s \neq t$. The CPS model indicates incomplete symmetry of cumulative probabilities for part of the symmetric pairs $\left\{G_{i j}, G_{j i}\right\}$. Note that the CPS model has a different structure from the PS model.

Consider the artificial probability tables in Table 1 . We can see that there is a structure of the PS model in Table 1(a), while there is not that of the PS model in Table 1(b). Tables 1(c) and 1(d) are the cumulative probability tables calculated from Tables 1(a) and 1(b), respectively. We can also see that there is not structure of the CPS model in Table 1(c) (and Table 1(a)), while there is that of the CPS model in Table 1(d) (and Table 1(b)). Therefore, the CPS model is not equivalent to the PS model. 
Table 1. Artificial cell probability tables and cumulative probability tables: the cumulative probabilities in Tables 1(c)-(d) are calculated from Tables 1(a)-(b), respectively

(a)

\begin{tabular}{llll}
\hline 0.140 & 0.017 & 0.033 & 0.018 \\
0.017 & 0.141 & 0.004 & 0.018 \\
0.066 & 0.016 & 0.140 & 0.015 \\
0.054 & 0.090 & 0.090 & 0.141 \\
\hline
\end{tabular}

(b)

\begin{tabular}{llll}
\hline 0.155 & 0.019 & 0.005 & 0.001 \\
0.001 & 0.150 & 0.050 & 0.004 \\
0.020 & 0.200 & 0.150 & 0.015 \\
0.004 & 0.016 & 0.060 & 0.150 \\
\hline
\end{tabular}

(c) Cumulative probabilities for Table 1(a)

\begin{tabular}{llll}
\hline & 0.068 & 0.051 & 0.018 \\
0.137 & & 0.073 & 0.036 \\
0.120 & 0.226 & & 0.051 \\
0.054 & 0.144 & 0.234 & \\
\hline
\end{tabular}

(d) Cumulative probabilities for Table 1(b)

\begin{tabular}{llll}
\hline & 0.025 & 0.006 & 0.001 \\
0.025 & & 0.060 & 0.005 \\
0.024 & 0.240 & & 0.020 \\
0.004 & 0.020 & 0.080 & \\
\hline
\end{tabular}




\subsection{Measure}

For an $r \times r$ contingency table with ordered categories, assume that $\left\{G_{i j}+G_{j i} \neq 0\right\}$ (namely, $p_{1 r}+p_{r 1} \neq 0$ ). We propose the measure as

$$
\gamma^{(\lambda)}=\prod_{i=1}^{r-1} \prod_{j=i+1}^{r}\left[\gamma_{i j}^{(\lambda)}\right]^{\left(G_{i j}^{*}+G_{j i}^{*}\right)} \quad(\lambda>-1)
$$

where

$$
\begin{aligned}
G_{i j}^{*} & =\frac{G_{i j}}{\Delta} \quad(i \neq j), \quad \Delta=\sum_{s \neq t} G_{s t}, \\
\gamma_{i j}^{(\lambda)} & =1-\frac{\lambda 2^{\lambda}}{2^{\lambda}-1} I_{i j}^{(\lambda)} \\
I_{i j}^{(\lambda)} & =\frac{1}{\lambda}\left(1-\left(G_{i j}^{c}\right)^{\lambda+1}-\left(G_{j i}^{c}\right)^{\lambda+1}\right) \\
G_{i j}^{c} & =\frac{G_{i j}}{G_{i j}+G_{j i}} \quad(i \neq j) .
\end{aligned}
$$

The value at $\lambda=0$ is taken to be the limit as $\lambda \rightarrow 0$. Namely, it is defined by

$$
\gamma^{(0)}=\prod_{i=1}^{r-1} \prod_{j=i+1}^{r}\left[\gamma_{i j}^{(0)}\right]^{\left(G_{i j}^{*}+G_{j i}^{*}\right)}
$$

where

$$
\begin{aligned}
& \gamma_{i j}^{(0)}=1-\frac{1}{\log 2} I_{i j}^{(0)}, \\
& I_{i j}^{(0)}=-G_{i j}^{c} \log G_{i j}^{c}-G_{j i}^{c} \log G_{j i}^{c} .
\end{aligned}
$$

The measure $\gamma^{(\lambda)}$ can be seen as the weighted geometric mean of diversity index $I_{i j}^{(\lambda)}$, which includes the Shannon entropy $I_{i j}^{(0)}$. This 
measure satisfies the following properties for any $\lambda(>-1)$ : (i) $\gamma^{(\lambda)}$ must lie between 0 and 1, (ii) $\gamma^{(\lambda)}$ takes the minimum value 0 if and only if the CPS model holds, (iii) $\gamma^{(\lambda)}$ takes the maximum value 1 if and only if the degree of departure from the CPS model is largest in the sense that $\left\{G_{i j}^{c}=1\right\}\left(\right.$ then $\left.\left\{G_{j i}^{c}=0\right\}\right)$ or $\left\{G_{j i}^{c}=1\right\}\left(\right.$ then $\left.\left\{G_{i j}^{c}=0\right\}\right)$ for all $i<j$.

The largest departure indicates complete asymmetry such that the cumulative probabilities in one side of upper-right or lower-left triangle of the table are all $0 \mathrm{~s}$ (then those in the reverse side are not all 0 s from assumption).

The measure $\gamma^{(\lambda)}$ is not invariant under arbitrary same permutations of row and column categories except for the reverse order, while the measure $\Phi^{(\lambda)}$ is invariant under arbitrary same permutations of the categories. Therefore, the measure $\gamma^{(\lambda)}$ is appropriate for a square contingency table with ordered categories because it depends on the order of categories in the table.

\section{Approximate Confidence Interval of Measure}

Let $n_{i j}$ denote the observed frequency in the $i$-th row and $j$-th column of the table $(i=1, \ldots, r ; j=1, \ldots, r)$, and let $n$ denote the total number of observations, i.e., $n=\sum \sum n_{i j}$. Assuming that a multinomial distribution applies to the $r \times r$ table, we shall consider an approximate standard error and large-sample confidence interval for the measure $\gamma^{(\lambda)}$. The estimate of measure, denoted by $\hat{\gamma}^{(\lambda)}$, is given by $\gamma^{(\lambda)}$ with $\left\{p_{i j}\right\}$ replaced by $\left\{\hat{p}_{i j}\right\}$, where $\hat{p}_{i j}=n_{i j} / n$. Using the delta method (Agresti [1], p. 587), $\sqrt{n}\left(\hat{\gamma}^{(\lambda)}-\gamma^{(\lambda)}\right)$ asymptotically $(n \rightarrow \infty)$ has a normal distribution with mean 0 and variance $\sigma^{2}\left[\gamma^{(\lambda)}\right]$, where 


$$
\sigma^{2}\left[\gamma^{(\lambda)}\right]=\left(\frac{\gamma^{(\lambda)}}{\Delta}\right)^{2}\left[\sum_{\substack{s=1 \\ t \\ t \neq 1}}^{r} \sum_{s t}^{r} p_{s t}\left(\omega_{s t}^{(\lambda)}\right)^{2}-\left(\sum_{\substack{s=1 \\ t}}^{r} \sum_{\substack{t=1 \\ t \neq s}}^{r} p_{s t} \omega_{s t}^{(\lambda)}\right)^{2}\right] \quad(\lambda>-1)
$$

with

$$
\begin{aligned}
& \omega_{s t}^{(\lambda)}=-d_{s t} \log \gamma^{(\lambda)}+\xi_{s t}^{(\lambda)}, \\
& d_{s t}=\frac{1}{2}\left((t-s)^{2}+|t-s|\right), \\
& \left(\sum_{s \leq i<j \leq t} \sum_{j}\left(\log \gamma_{i j}^{(\lambda)}+\frac{2^{\lambda}(\lambda+1) G_{j i}^{c}}{\left(2^{\lambda}-1\right) \gamma_{i j}^{(\lambda)}}\left(\left(G_{i j}^{c}\right)^{\lambda}-\left(G_{j i}^{c}\right)^{\lambda}\right)\right) \quad(\lambda \neq 0 ; s<t),\right. \\
& \xi_{s t}^{(\lambda)}= \begin{cases}\sum_{t \leq i<j \leq s} \sum_{l}\left(\log \gamma_{i j}^{(\lambda)}+\frac{2^{\lambda}(\lambda+1) G_{i j}^{c}}{\left(2^{\lambda}-1\right) \gamma_{i j}^{(\lambda)}}\left(\left(G_{j i}^{c}\right)^{\lambda}-\left(G_{i j}^{c}\right)^{\lambda}\right)\right) & (\lambda \neq 0 ; s>t), \\
\sum_{s \leq i<j \leq t} \sum_{j \leq}\left(\log \gamma_{i j}^{(0)}+\frac{G_{j i}^{c}}{(\log 2) \gamma_{i j}^{(0)}}\left(\log G_{i j}^{c}-\log G_{j i}^{c}\right)\right) & (\lambda=0 ; s<t), \\
\sum_{t \leq i<j \leq s} \sum_{i j}\left(\log \gamma_{i j}^{(0)}+\frac{G_{i j}^{c}}{(\log 2) \gamma_{i j}^{(0)}}\left(\log G_{j i}^{c}-\log G_{i j}^{c}\right)\right) & (\lambda=0 ; s>t) .\end{cases}
\end{aligned}
$$

This asymptotic distribution is applicable only when $0<\gamma^{(\lambda)}<1$ because $\sigma^{2}\left[\gamma^{(\lambda)}\right]=0$ when $\gamma^{(\lambda)}=0$ and 1. Let $\hat{\sigma}^{2}\left[\gamma^{(\lambda)}\right]$ denote $\sigma^{2}\left[\gamma^{(\lambda)}\right]$ with $\left\{p_{i j}\right\}$ replaced by $\left\{\hat{p}_{i j}\right\}$. Then $\hat{\sigma}\left[\gamma^{(\lambda)}\right] / \sqrt{n}$ is the estimated approximate standard error for $\hat{\gamma}^{(\lambda)}$, and $\left.\hat{\gamma}^{(\lambda)} \pm z_{\alpha / 2} \hat{\sigma}^{(\lambda)}\right] / \sqrt{n}$ is the approximate $100(1-\alpha)$ percent confidence interval for $\gamma^{(\lambda)}$, where $z_{\alpha / 2}$ is the upper $(\alpha / 2)$-th quantile of standard normal distribution.

The values in the confidence interval may be not bounded by $(0,1)$. Thus we also give another one for measure $\gamma^{(\lambda)}$ using $\log (-\log )$ transformation. Let $\theta^{(\lambda)}=\log \left[-\log \left(\gamma^{(\lambda)}\right)\right]$, and let $\hat{\theta}^{(\lambda)}$ denote the 
sample version of $\theta^{(\lambda)}$ which is given by $\theta^{(\lambda)}$ with $\left\{p_{i j}\right\}$ replaced by $\left\{\hat{p}_{i j}\right\}$. Using the delta method, $\sqrt{n}\left(\hat{\theta}^{(\lambda)}-\theta^{(\lambda)}\right)$ asymptotically $(n \rightarrow \infty)$ has a normal distribution with mean 0 and variance $\sigma^{2}\left[\theta^{(\lambda)}\right]$, where

$$
\sigma^{2}\left[\theta^{(\lambda)}\right]=\left(\frac{\sigma\left[\gamma^{(\lambda)}\right]}{\gamma^{(\lambda)} \log \gamma^{(\lambda)}}\right)^{2} \quad(\lambda>-1) .
$$

Therefore another version of approximate confidence interval using the $\log (-\log ) \quad$ transformation is then given by $\exp \left(-\exp \left(\hat{\theta}^{(\lambda)} \mp\right.\right.$ $\left.z_{\alpha / 2} \hat{\sigma}\left[\theta^{(\lambda)}\right] / \sqrt{n}\right)$ ), where $\hat{\sigma}\left[\theta^{(\lambda)}\right]$ is given by $\sigma\left[\theta^{(\lambda)}\right]$ with $\left\{p_{i j}\right\}$ replaced by $\left\{\hat{p}_{i j}\right\}$. We note that the asymptotic confidence limits are bounded by $(0,1)$ (Lachin [5], p.17).

\section{Examples}

Consider the data in Table 2 taken from Hashimoto [4]. These data describe the cross-classifications of father's and his son's occupational status categories in Japan which were examined in 1955, 1965 and 1975. The smaller category number means a higher status.

Table 3 gives the estimated values of measure $\gamma^{(\lambda)}$ applied to each of the data in Tables 2(a)-(c). It also gives the estimated approximate standard errors and the two kinds of approximate 95\% confidence intervals of the measure. We shall compare the degrees of departure from a structure of the CPS model toward complete asymmetry for cumulative probabilities among the data in Tables 2(a)-(c) using the proposed measure $\gamma^{(\lambda)}$. For any one of the two confidence intervals and any $\lambda(>-1)$, we can see that the values in a confidence interval for each of the data in Tables 2(b)-(c) are greater than those for the data in Table 2(a). Thus it is inferred that the degrees of departure from the CPS model for Japanese father's and his son's occupational status are larger in 1965 and in 1975 than in 1955. 
Table 2. Occupational status for Japanese father-son pairs (Hashimoto [4], p. 151)

(a) Estimated in 1955

\begin{tabular}{ccccccc}
\hline & \multicolumn{5}{c}{ Son's status } \\
\cline { 2 - 5 } Father's status & $(1)$ & $(2)$ & $(3)$ & $(4)$ & $(5)$ & Total \\
\hline$(1)$ & 39 & 39 & 39 & 57 & 23 & 197 \\
$(2)$ & 12 & 78 & 23 & 23 & 37 & 173 \\
$(3)$ & 6 & 16 & 78 & 23 & 20 & 143 \\
$(4)$ & 18 & 80 & 79 & 126 & 31 & 334 \\
$(5)$ & 28 & 106 & 136 & 122 & 628 & 1020 \\
\hline Total & 103 & 319 & 355 & 351 & 739 & 1867 \\
\hline
\end{tabular}

(b) Estimated in 1965

\begin{tabular}{ccccccc}
\hline & \multicolumn{5}{c}{ Son's status } \\
\cline { 2 - 5 } Father's status & $(1)$ & $(2)$ & $(3)$ & $(4)$ & $(5)$ & Total \\
\hline$(1)$ & 64 & 51 & 26 & 30 & 6 & 177 \\
$(2)$ & 18 & 102 & 51 & 18 & 6 & 195 \\
$(3)$ & 6 & 48 & 138 & 24 & 11 & 227 \\
$(4)$ & 35 & 82 & 107 & 112 & 13 & 349 \\
$(5)$ & 30 & 140 & 279 & 106 & 306 & 861 \\
\hline Total & 153 & 423 & 601 & 290 & 342 & 1809 \\
\hline
\end{tabular}

(c) Estimated in 1975

\begin{tabular}{ccccccc}
\hline & \multicolumn{5}{c}{ Son's status } & \\
\cline { 2 - 5 } Father's status & $(1)$ & $(2)$ & $(3)$ & $(4)$ & $(5)$ & Total \\
\hline$(1)$ & 29 & 43 & 25 & 31 & 4 & 132 \\
$(2)$ & 23 & 159 & 89 & 38 & 14 & 323 \\
$(3)$ & 11 & 69 & 184 & 34 & 10 & 308 \\
$(4)$ & 42 & 147 & 148 & 184 & 17 & 538 \\
$(5)$ & 42 & 176 & 377 & 114 & 298 & 1007 \\
\hline Total & 147 & 594 & 823 & 401 & 343 & 2308 \\
\hline
\end{tabular}

Note: Status (1) is Capitalist; (2) New middle; (3) Working; (4) Selfemployed; and (5) Farming. 
Table 3. The estimates of $\gamma^{(\lambda)}, \hat{\gamma}^{(\lambda)}$, estimated approximate standard errors (SE) of $\hat{\gamma}^{(\lambda)}$, approximate 95\% confidence intervals (CI) of $\gamma^{(\lambda)}$ and CIs using the $\log (-\log )$ transformation $(\mathrm{CI}(\log -\log ))$, applied to each of the data in Tables 2(a)-(c)

(a) For Table 2(a)

\begin{tabular}{ccccc}
\hline$\lambda$ & $\hat{\gamma}^{(\lambda)}$ & $\mathrm{SE}$ & $\mathrm{CI}$ & $\mathrm{CI}(\log -\log )$ \\
\hline-0.5 & 0.050 & 0.012 & $(0.027,0.073)$ & $(0.030,0.076)$ \\
0.0 & 0.082 & 0.019 & $(0.045,0.119)$ & $(0.050,0.124)$ \\
0.5 & 0.101 & 0.023 & $(0.056,0.147)$ & $(0.062,0.152)$ \\
1.0 & 0.111 & 0.025 & $(0.061,0.160)$ & $(0.067,0.166)$ \\
1.5 & 0.113 & 0.026 & $(0.063,0.163)$ & $(0.069,0.169)$ \\
2.0 & 0.111 & 0.025 & $(0.061,0.160)$ & $(0.067,0.166)$ \\
\hline
\end{tabular}

(b) For Table 2(b)

\begin{tabular}{ccccc}
\hline$\lambda$ & $\hat{\gamma}^{(\lambda)}$ & $\mathrm{SE}$ & $\mathrm{CI}$ & $\mathrm{CI}(\log -\log )$ \\
\hline-0.5 & 0.175 & 0.025 & $(0.126,0.224)$ & $(0.129,0.227)$ \\
0.0 & 0.264 & 0.036 & $(0.194,0.334)$ & $(0.197,0.336)$ \\
0.5 & 0.308 & 0.040 & $(0.229,0.387)$ & $(0.231,0.387)$ \\
1.0 & 0.326 & 0.042 & $(0.244,0.409)$ & $(0.246,0.409)$ \\
1.5 & 0.331 & 0.042 & $(0.248,0.414)$ & $(0.250,0.414)$ \\
2.0 & 0.326 & 0.042 & $(0.244,0.409)$ & $(0.246,0.409)$ \\
\hline
\end{tabular}

(c) For Table 2(c)

\begin{tabular}{ccccc}
\hline$\lambda$ & $\hat{\gamma}^{(\lambda)}$ & $\mathrm{SE}$ & $\mathrm{CI}$ & $\mathrm{CI}(\log -\log )$ \\
\hline-0.5 & 0.193 & 0.033 & $(0.128,0.259)$ & $(0.133,0.263)$ \\
0.0 & 0.291 & 0.049 & $(0.196,0.386)$ & $(0.200,0.388)$ \\
0.5 & 0.339 & 0.056 & $(0.230,0.447)$ & $(0.233,0.447)$ \\
1.0 & 0.359 & 0.058 & $(0.245,0.473)$ & $(0.247,0.472)$ \\
1.5 & 0.364 & 0.059 & $(0.248,0.479)$ & $(0.251,0.478)$ \\
2.0 & 0.359 & 0.058 & $(0.245,0.473)$ & $(0.247,0.472)$ \\
\hline
\end{tabular}




\section{Discussion}

Consider the $4 \times 4$ artificial cell and cumulative probability tables given in Tables 4 and 5. The cumulative probabilities given in Table 5 can arise with the cell probabilities given in Table 4. Table 5(a) has a structure of CPS with $G_{12}=G_{21}=0.025$. For each of Tables 5(b)-(e), the value of $G_{k l}$ for any $k \neq l$ with $(k, l) \neq(1,2)$ equals the value of $G_{k l}$ in Table 5(a). The ratio $G_{12} / G_{21}$ varies for Tables 5(a)-(e): 1.0 for Table 5(a), 2.0 for Table 5(b), 3.0 for Table 5(c), 4.0 for Table 5(d), and 5.0 for Table 5(e). Thus, it is natural to consider that the degree of departure from the CPS model increases in the order of Tables 5(a)-(e). The cumulative probabilities given in Table 5(f) shows complete asymmetry in the sense that cumulative probabilities in upper-right triangle cells are all 0s.

Table 4. Artificial cell probability tables

(a)

\begin{tabular}{llll}
\hline 0.155 & 0.019 & 0.005 & 0.001 \\
0.001 & 0.150 & 0.050 & 0.004 \\
0.020 & 0.200 & 0.150 & 0.015 \\
0.004 & 0.016 & 0.060 & 0.150 \\
\hline
\end{tabular}

(b)

\begin{tabular}{llll}
\hline 0.145 & 0.044 & 0.005 & 0.001 \\
0.001 & 0.145 & 0.050 & 0.004 \\
0.020 & 0.200 & 0.145 & 0.015 \\
0.004 & 0.016 & 0.060 & 0.145 \\
\hline
\end{tabular}

(c)

\begin{tabular}{llll}
\hline 0.138 & 0.069 & 0.005 & 0.001 \\
0.001 & 0.139 & 0.050 & 0.004 \\
0.020 & 0.200 & 0.139 & 0.015 \\
0.004 & 0.016 & 0.060 & 0.139 \\
\hline
\end{tabular}


Table 4. (Continued)

(d)

\begin{tabular}{cccc}
\hline 0.135 & 0.094 & 0.005 & 0.001 \\
0.001 & 0.135 & 0.050 & 0.004 \\
0.020 & 0.200 & 0.135 & 0.015 \\
0.004 & 0.016 & 0.060 & 0.125 \\
\hline \multicolumn{5}{c}{$(\mathrm{e})$} \\
\hline 0.126 & 0.119 & 0.005 & 0.001 \\
0.001 & 0.126 & 0.050 & 0.004 \\
0.020 & 0.200 & 0.127 & 0.015 \\
0.004 & 0.016 & 0.060 & 0.126 \\
\hline \multicolumn{5}{c}{$(\mathrm{f})$} \\
\hline 0.175 & 0 & 0 & 0 \\
0.001 & 0.174 & 0 & 0 \\
0.020 & 0.200 & 0.175 & 0 \\
0.004 & 0.016 & 0.060 & 0.175 \\
\hline
\end{tabular}


Table 5. Artificial cumulative probability tables: the cumulative probabilities in Tables 5(a)-(f) are calculated from Tables 4(a)-(f), respectively

(a)

\begin{tabular}{llll}
\hline & 0.025 & 0.006 & 0.001 \\
0.025 & & 0.060 & 0.005 \\
0.024 & 0.240 & & 0.020 \\
0.004 & 0.020 & 0.080 & \\
\hline
\end{tabular}

(b)

\begin{tabular}{llll}
\hline & 0.050 & 0.006 & 0.001 \\
0.025 & & 0.060 & 0.005 \\
0.024 & 0.240 & & 0.020 \\
0.004 & 0.020 & 0.080 & \\
\hline
\end{tabular}

(c)

\begin{tabular}{llll}
\hline & 0.075 & 0.006 & 0.001 \\
0.025 & & 0.060 & 0.005 \\
0.024 & 0.240 & & 0.020 \\
0.004 & 0.020 & 0.080 & \\
\hline
\end{tabular}

(d)

\begin{tabular}{|c|c|c|c|}
\hline & 0.100 & 0.006 & 0.001 \\
\hline 0.025 & & 0.060 & 0.005 \\
\hline 0.024 & 0.240 & & 0.020 \\
\hline 0.004 & 0.020 & 0.080 & \\
\hline \multicolumn{4}{|c|}{ (e) } \\
\hline & 0.125 & 0.006 & 0.001 \\
\hline 0.025 & & 0.060 & 0.005 \\
\hline 0.024 & 0.240 & & 0.020 \\
\hline 0.004 & 0.020 & 0.080 & \\
\hline
\end{tabular}


Table 5. (Continued)

\begin{tabular}{cccc}
\multicolumn{5}{c}{$(\mathrm{f})$} \\
\hline & 0 & 0 & 0 \\
0.025 & & 0 & 0 \\
0.024 & 0.240 & & 0 \\
0.004 & 0.020 & 0.080 & \\
\hline
\end{tabular}

Table 6 gives the values of measure $\gamma^{(\lambda)}$ applied to each of Tables 5(a)-(f). As seen in Table 6, we see that (1) the value of $\gamma^{(\lambda)}$ for Table 5(a) equals 0 , (2) for any fixed $\lambda$, the value of $\gamma^{(\lambda)}$ increases as the ratio $G_{12} / G_{21}$ increases, and (3) the value of $\gamma^{(\lambda)}$ for Table 5(f) equals 1. Therefore, the measure $\gamma^{(\lambda)}$ would be appropriate to represent the degree of departure from the CPS model.

Table 6. Values of $\gamma^{(\lambda)}$ applied to each of Tables 5(a)-(f)

\begin{tabular}{cccc}
\hline Applied & \multicolumn{3}{c}{$\lambda$} \\
\cline { 2 - 4 } tables & 0 & 0.5 & 1.0 \\
\hline Table 5(a) & 0.000 & 0.000 & 0.000 \\
Table 5(b) & 0.234 & 0.283 & 0.305 \\
Table 5(c) & 0.259 & 0.313 & 0.337 \\
Table 5(d) & 0.278 & 0.334 & 0.360 \\
Table 5(e) & 0.294 & 0.353 & 0.379 \\
Table 5(f) & 1.000 & 1.000 & 1.000 \\
\hline
\end{tabular}

Consider the artificial cell probability table in Table 7(a), and the modified table which is obtained by interchanging categories 2 and 3 in Table 7(b). Tables 7(c)-(d) give the value of measures $\Phi^{(\lambda)}$ and $\gamma^{(\lambda)}$ applied to each of Tables 7(a)-(b), respectively. From Tables 7(c)-(d), we can see that the value of $\gamma^{(\lambda)}$ for Table $7(\mathrm{a})$ is different from that for Table 7(b), while the value of $\Phi^{(\lambda)}$ for Table 7(a) is the same as that for Table 7(b). It is because that the measure $\gamma^{(\lambda)}$ depends on the order of categories of the table unlike the measure $\Phi^{(\lambda)}$. 
Table 7. (a) Artificial cell probability table and (b) the modified table interchanging categories 2 and 3, and (c)-(d) values of $\Phi^{(\lambda)}$ and $\gamma^{(\lambda)}$ applied to each of Tables 7(a)-(b)

(a)

\begin{tabular}{llll}
\hline 0.090 & 0.027 & 0.060 & 0.010 \\
0.055 & 0.160 & 0.072 & 0.090 \\
0.049 & 0.062 & 0.140 & 0.035 \\
0.015 & 0.066 & 0.029 & 0.040 \\
\hline
\end{tabular}

(b)

\begin{tabular}{llll}
\hline 0.090 & 0.060 & 0.027 & 0.010 \\
0.049 & 0.140 & 0.062 & 0.035 \\
0.055 & 0.072 & 0.160 & 0.090 \\
0.015 & 0.029 & 0.066 & 0.040 \\
\hline
\end{tabular}

(c) For Table 7(a)

\begin{tabular}{ccc}
$\lambda$ & $\Phi^{(\lambda)}$ & $\gamma^{(\lambda)}$ \\
\hline 0.0 & 0.012 & 0.006 \\
0.5 & 0.015 & 0.008 \\
1.0 & 0.017 & 0.009 \\
\hline
\end{tabular}

(d) For Table 7(b)

\begin{tabular}{ccc}
\hline$\lambda$ & $\Phi^{(\lambda)}$ & $\gamma^{(\lambda)}$ \\
\hline 0.0 & 0.012 & 0.007 \\
0.5 & 0.015 & 0.009 \\
1.0 & 0.017 & 0.010 \\
\hline
\end{tabular}




\section{Concluding Remarks}

For a square contingency table with ordered categories, we have considered the CPS model which indicates partial symmetry of cumulative probabilities for part of the symmetric pairs of cumulative probabilities.

We have proposed the measure to express the degree of departure from the CPS model. The proposed measure $\gamma^{(\lambda)}$ enables us to see how far cumulative probabilities are distant from those with the CPS model toward complete asymmetry because the measure has property that it increases as the degree of departure from the CPS model increases. The measure $\gamma^{(\lambda)}$ is also useful for comparing the degrees of departure from the CPS model between different tables (see examples). Also, the measure $\gamma^{(\lambda)}$ is appropriate for the square table with ordered categories.

\section{References}

[1] A. Agresti, Categorical Data Analysis, 3rd Edition, John Wiley and Sons, Hoboken, New Jersey, 2013.

[2] Y. M. M. Bishop, S. E. Fienberg and P. W. Holland, Discrete Multivariate Analysis: Theory and Practice, MIT Press, Cambridge, 1975.

DOI: https://doi.org/10.1007/978-0-387-72806-3

[3] A. H. Bowker, A test for symmetry in contingency tables, Journal of the American Statistical Association 43(244) (1948), 572-574.

[4] K. Hashimoto, Gendai Nihon no Kaikyu Kozo (Class Structure in Modern Japan: Theory, Method and Quantitative Analysis), Toshindo Press, Tokyo (in Japanese), 1999.

[5] J. M. Lachin, Biostatistical Methods: The Assessment of Relative Risks, 2nd Edition, John Wiley and Sons, Hoboken, New Jersey, 2011.

[6] G. P. Patil and C. Taillie, Diversity as a concept and its measurement, Journal of the American Statistical Association 77(379) (1982), 548-561.

[7] Y. Saigusa, K. Tahata and S. Tomizawa, Measure of departure from partial symmetry for square contingency tables, Journal of Mathematics and Statistics 12(3) (2016), 152-156.

DOI: https://doi.org/10.3844/jmssp.2016.152.156 
[8] S. Tomizawa, N. Miyamoto and Y. Hatanaka, Measure of asymmetry for square contingency tables having ordered categories, The Australian and New Zealand Journal of Statistics 43(3) (2001), 335-349.

DOI: https://doi.org/10.1111/1467-842X.00180

[9] S. Tomizawa, T. Seo and H. Yamamoto, Power-divergence-type measure of departure from symmetry for square contingency tables that have nominal categories, Journal of Applied Statistics 25(3) (1998), 387-398.

DOI: https://doi.org/10.1080/02664769823115 